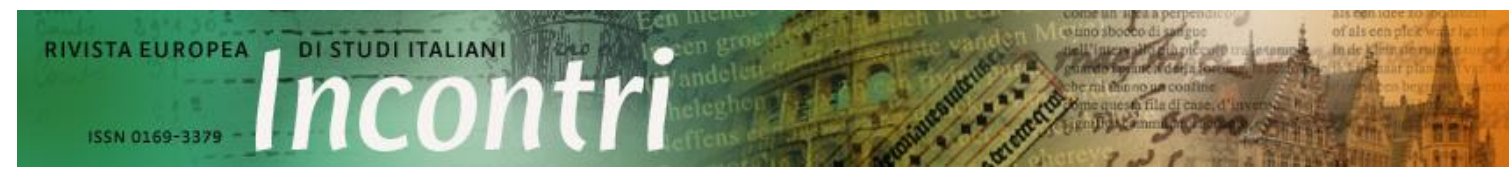

Anno 35, 2020 / Fascicolo 1/ p. 59-67 - unw.rivista-incontri.nl - http://doi.org/10.18352/incontri.10334

(C) The author(s) - Content is licensed under a Creative Commons Attribution 3.0 Unported License Publisher: Werkgroep Italië Studies, supported by Utrecht University Library Open Access Journals

\title{
Leonardo's folio 735 of the Codex Atlanticus How Leonardo discovered more solids than those mentioned in De Divina Proportione
}

\author{
Dirk Huylebrouck \& Angelo Mingarelli
}

\section{Introduction}

One of the most famous early modern books on mathematics, Luca Pacioli's De Divina Proportione, contains a series of well-known drawings of polyhedra attributed to Leonardo da Vinci. The book and the beauty of its illustrations created a true revival of the interest for polyhedra, not only among mathematicians but also in the eyes of artists. Pacioli begins the printed version of his De Divina Proportione by a letter addressed to Piero Soderini (1450-1522), the gonfaloniere della giustizia a vita or "standard bearer of justice for life", praising the fact that the drawings were made "by the hands of our Leonardo da Vinci so that one could understand them better with the eyes'. ${ }^{1}$ This printed version appeared in 1509, while the two (or, perhaps, three) manuscript versions were already finished in $1498 .^{2}$ The book shows prisms, pyramids, cylinders, spheres, the five Platonic solids and six of the thirteen Archimedean solids. ${ }^{3}$ Pacioli often describes four versions of the solids, all of which have their visual representation in the drawings: hollow and solid (i.e. polyhedra in skeletal form, consisting of a hollow structure in which almost all edges are visible versus nontransparent polyhedra with opaque faces) and with or without pyramids on each face (i.e. "elevated" or "plane" polyhedra). The treatise documents the collaboration between Leonardo and his friend Luca Pacioli, generally considered to be his teacher in mathematical issues. During his stay in Milan at the Sforza court, Leonardo probably experimented intensively with visual representations of solids, as appears from drawings collected in his Codex Atlanticus.

\footnotetext{
${ }^{1}$ L. Pacioli, De Divina Proportione, Venezia, Paganino Paganini, 1509, p. A ii. Our translation.

2 L. Pacioli, Divine Proportion, G. Duchesne \& M. Giraud (French translation), Paris, Librairie du Compagnonnage, 1980, p. 29.

${ }^{3}$ Some distinguish a 14th Archimedean solid, the pseudo-rhombicuboctahedron (first described in 1905), which has less symmetry and is therefore generally not included in the list of Archimedeans solids. This solid plays a role in another discussion related to the polyhedra represented by Leonardo (see D. Huylebrouck, 'Lost in Triangulation: Leonardo da Vinci's Mathematical Slip-Up', in: Scientific American, 4 (2011), https://www.scientificamerican.com/article/davinci-mathematical-slip-up/, consulted on 9 June 2020).
} 


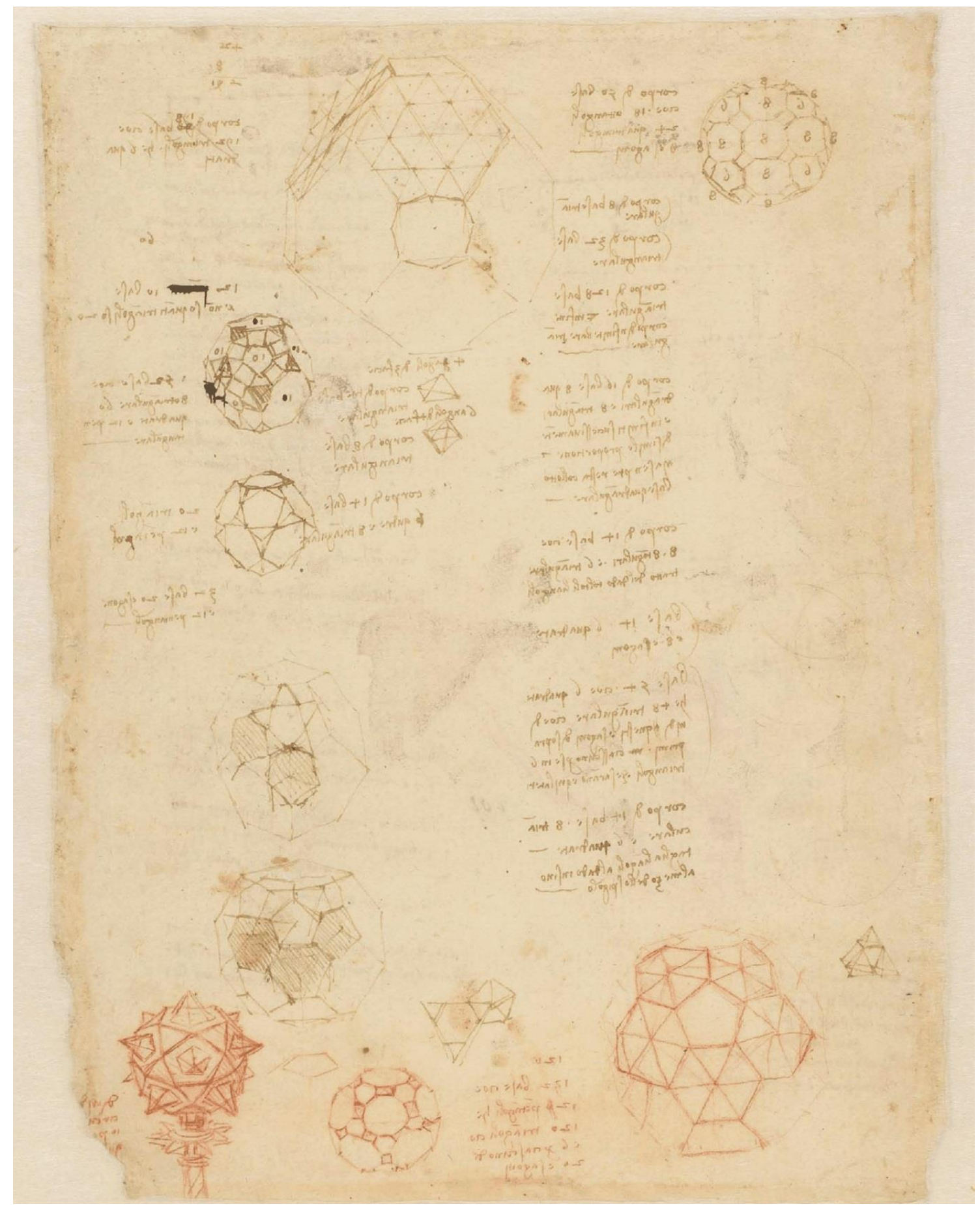

Fig. 1: Leonardo's folio 735 from his Codex Atlanticus.

Strangely enough, folio 735 verso of Leonardo's Codex Atlanticus seems to show two more Archimedean solids that do not appear in Pacioli's account on all of the then known polyhedral (Figure 1). Carlo Pedretti dates the folio from 1495-7, ${ }^{4}$ which fits well with the time when Pacioli and Leonardo came to Milan, at the end of 1495 or in

\footnotetext{
${ }^{4}$ C. Pedretti, 'The Codex Atlanticus of Leonardo da Vinci: A Catalogue of Its Newly Restored Sheets', 2
} vols., New York, Johnson Reprint Corporation, 1978-1979. 
1496. ${ }^{5}$ At the court of Ludovico Maria Sforza they worked together and thus the drawings on this folio could have been inspired by the other polyhedra in De Divina Proportione. However, though Pacioli wanted to give an account of all of the then known polyhedra, some of the solids Leonardo discovered on folio 735 were not included in his treatise. This article aims to shed light on these discoveries by offering an interpretation of Leonardo's drawings and annotations.

The motivation for this article arose from a quite recent description of this folio. ${ }^{6}$ It starts as follows: 'Various studies of geometric solids. This folio is related to the treatise by Luca Pacioli De Divina Proportione, dedicated to the five 'regular bodies' that Plato had cited as symbols of the natural elements in Timaeus: the cube for earth, the pyramid for fire'. ${ }^{7}$ It goes on describing Leonardo's predilection for the Platonic solids and concludes: 'In the poetic fiction the five geometric solids are given voice to sing the praises of the fruit of intellectual delectation, the purpose of which is to discover the philosophic reasoning contained in them'. These comments give no information about the contents of the folio as Archimedean and more general solids are the topic of this folio, not the Platonic (not a single Platonic solid is represented on the folio). The present paper tries to propose a more judicious analysis of this folio, as was done previously for other drawings by Leonardo. ${ }^{8}$

The thirteen Archimedean solids are the (1) truncated tetrahedron, the (2) truncated icosahedron, the (3) truncated octahedron, the (4) cuboctahedron (called truncated cube by Pacioli; it has 8 triangular and 6 square faces), the (5) icosidodecahedron and the (6) rhombicuboctahedron, the (7) truncated dodecahedron, the (8) truncated icosidodecahedron, the (9) truncated cuboctahedron, the (10) truncated cube (having 6 octagonal and 8 triangular faces), the (11) rhombicosidodecahedron, the (12) snub cube and the (13) snub dodecahedron. Pacioli mentions polyhedra (1-6) in De Divina Proportione, but not (7-13). Yet, Leonardo does represent the (7) truncated dodecahedron and (8) truncated icosidodecahedron on his folio 735.

\section{The folio}

On the top of the folio there is a solid that looks like a truncated octahedron at first sight. Two faces are filled up with equilateral triangles. However, such a polyhedron cannot be a truncated octahedron, as three regular hexagons meet in one vertex at some point. The polyhedron given here in Figure 2 left resembles it, but the shaded hexagons in this figure aren't regular. It has 6 squares and 12 hexagons (of which 4 are irregular) and so the numbers given next to Leonardo's drawing - 8, 32 and 128 - do not seem to relate to it either. It remains unclear what Leonardo wanted to draw here, because if the depicted solid is formed by regular polygons, it clearly is impossible.

\footnotetext{
${ }^{5}$ Pedretti's periodization gives a better indication than the date given on the web page of the Biblioteca Ambrosiana, i.e. 1495: 'Il Codice Atlantico di Leonardo da Vinci': http://www.codexatlanticus.it/\#/Overview, consulted on 16 May 2019.

6 M. Versiero, Codex Atlanticus \#04. Leonardo, la politica e le allegorie / Leonardo, Politics and Allegories, Novara, D’Agostini, 2010, p. 140.

7 By 'the pyramid' the author may have referred to the tetrahedron, though these of course are no synonyms.

${ }^{8}$ See D. Huylebrouck, 'Lost in Enumeration: Leonardo da Vinci's Slip-ups in Arithmetic and Mechanics', in: The Mathematical Intelligencer, 34, 4 (2012), pp. 15-20; Id., 'Observations about Leonardo's drawings for Luca Pacioli', in: Journal of the British Society for the History of Mathematics, 30, 2 (2015), pp. 102112; Id., 'A Divine Error', Proceedings of the Bridges Conference 2015 on Mathematics, Music, Art, Architecture, Culture, Baltimore, 2015, pp. 93-98, and references reported therein (https://archive.bridgesmathart.org/2015/index.html, consulted on 15 June 2020).
} 
However, it is also true that the numbers 8,32 and 128 correspond to the number of triangles on a 'corpo di 8 basi triangulari', that is, 'a body with 8 triangular faces', or an octahedron. When each of its faces is triangulated by joining the mid-points of each of its sides, $8 \times 4=32$ smaller congruent triangles are obtained, that is, a 'corpo di 32 basi triangulari'. The next level of triangulations gives a 'corpo di 128 basi triangulari' $(32 \times 4=128)$, and this triangulation can be continued ad infinitum: 'infini corpi infini basi triangulari'. Thus, Leonardo would have written down these numbers as a reminder while triangulating the hexagonal faces of a regular octahedron.

Similarly, for the drawing on its right: in the centre there is a regular octagon, surrounded with squares and regular octagons. This implies that this part should be flat. However, on some faces Leonardo writes '6', in his typical mirror writing; it can therefore be suggested that he was exploring this shape and about to discover the truncated cuboctahedron - the Archimedean solid with 12 squares, 8 regular hexagons and 6 regular octagons. Yet, Leonardo describes the solid he was drawing in the text next to it: a solid with 50 faces, of which 18 are octagons, 24 are quadrangles, and 8 are hexagons. If three rings of 8 regular octagons cross perpendicularly, there are 18 octagons in all and what is left can be filled with 24 (irregular) quadrangles and 8 (irregular) hexagons (see Figure 2; the irregular quadrangles are grey and the irregular hexagons shaded). Thus, for this solid the number of faces corresponds exactly to the numbers given by Leonardo, but the solid is not an Archimedean one. Alternatively, Leonardo discovered the truncated rhombicuboctahedron: a non-Archimedean solid that indeed has 24 squares, 8 regular hexagons and 18 octagonal faces. Of the latter 6 are regular and 12 are irregular (see Figure 2; the 12 irregular octagons are grey). If this is the case, this would mean it is yet another polyhedron discovered by Leonardo and not included in Pacioli's book.
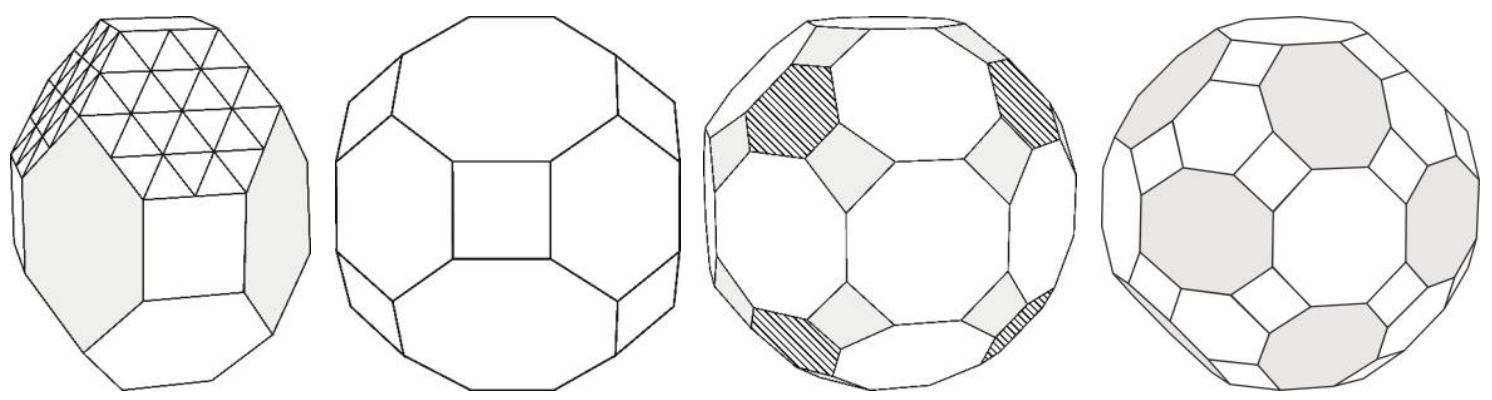

Fig. 2: Drawing similar to Leonardo's drawings on the top of the folio in the middle (left); the truncated cuboctahedron (middle left); drawings similar to Leonardo's on the top of the folio on the right: a polyhedron with regular octagons and irregular squares and hexagons (middle right), and the truncated rhombicuboctahedron, with irregular octagons (right).

Below this large "almost truncated" octahedron and on the left, Leonardo drew a polyhedron with the number ' 10 ' on several of its faces. Two of the faces have elevated parts formed by squares, triangles and a pentagon. In his mirror handwriting Leonardo describes it as follows: ' 12 [erasure that probably started with pentan, for pentagonal] - 10 figures and I don't know how many triangles there are' followed by '152 figures, that is, 80 triangular, 60 square [quadrati], and 12 pentagonal'. The 80 triangles are obtained by adding the original 20 triangles to the $3 \times 20=60$ additional triangles formed by erecting a triangular pyramid on each of the 20 original triangles. The 60 squares follow from the construction of 5 squares within each of the 12 octagons depicted by Leonardo in his drawing (thereby resulting in 5x12=60 squares). 
One could argue Leonardo forgot to subtract the original 20 triangles from the total of 80 triangles as 20 of them are covered by three additional ones in the augmentation of the truncated dodecahedron, but it was not uncommon to include the covered faces when a pyramid was constructed on them: Pacioli often did so too in his Divina Proportione, saying these covered faces 'can only be seen by the mind'. Figure 3 shows a reconstruction, as well as the same solid without the elevated parts: a truncated dodecahedron. And so, incidentally, Leonardo drew another solid not mentioned in Pacioli's De Divina Proportione.
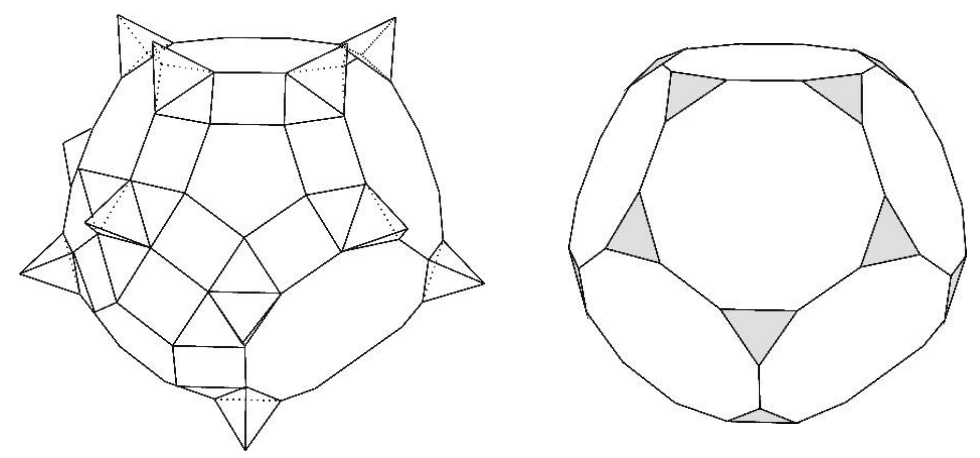

Fig. 3: Reconstruction of Leonardo's drawing on the left of the folio, second row (left); the underlying truncated dodecahedron (right).

It is possible Leonardo saw the truncated dodecahedron in a book by Piero della Francesca (1420-1492), Libellus de quinque corporibus regularibus ('On the Five Regular Bodies'). ${ }^{9}$ Actually, Leonardo draws it in exactly the same position (see Figure 4). This however is not a very convincing argument for stating Leonardo drew it after Piero's example, since it is plausible that anyone trying to draw a truncated dodecahedron would start by drawing a large decagon as a face frontal to the observer.

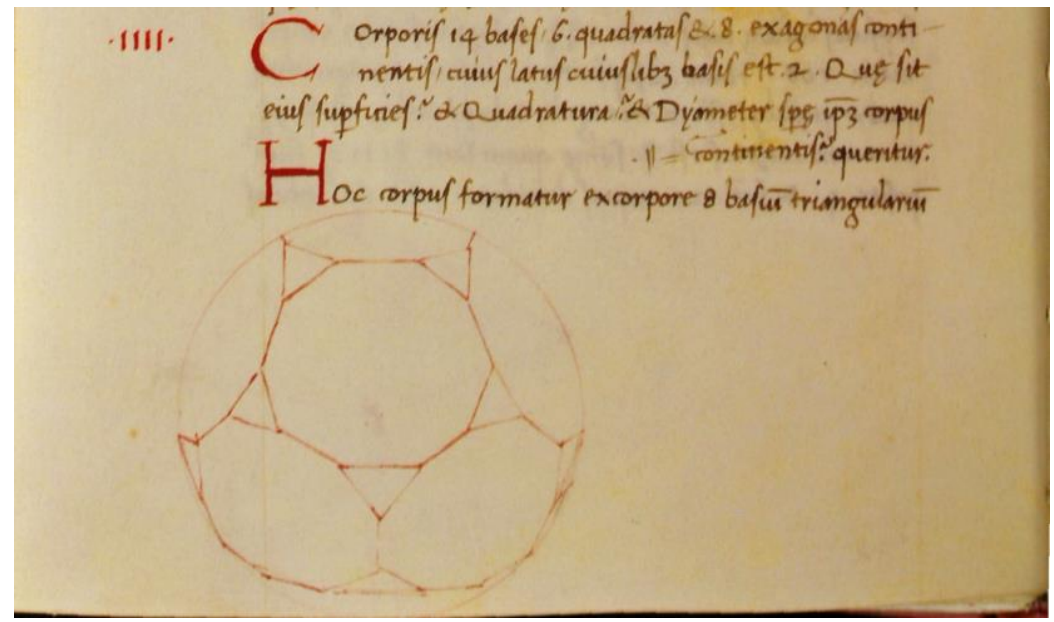

Fig. 4: Piero's drawing of the truncated dodecahedron. ${ }^{10}$

\footnotetext{
9 P. della Francesca, Libellus de quinque corporibus regularibus, F. di Teodoro (ed.), Firenze, Edizione nazionale degli scritti di Piero della Francesca, Firenze, Giunti, 1995.

10 Wikimedia Commons, consulted on 12 February 2020: https://commons.wikimedia.org/wiki/Libellus_ De_Quinque_Corporibus_Regularibus_de_Piero_della_Francesca.
} 
Note J.V. Field gives another interpretation of this drawing: 'it resembles the rhombicosidodecahedron, which was first illustrated by Kepler (1619). [...] Kepler presents this solid as analogous to the rhombicuboctahedron (first shown by Pacioli), and Leonardo may have conceived it in the same way, though the solid shown in his drawing is not possible in mathematical terms (unless some faces are not regular)' ${ }^{11}$ Field's interpretation might be explained by the fact that Leonardo didn't subdivide all of the decagons using triangles, squares and a pentagon. However, as we have seen, Leonardo's text confirms that this (incomplete) drawing is a polyhedron with 80 triangles, 60 squares and 12 pentagons.

There is a link though with the rhombicosidodecahedron which, by the way, does not appear in the Divina either. If Leonardo had continued subdividing the decagons with triangles, squares and pentagons, and if the triangular pyramids erected on the triangles between the decagons had been omitted, Leonardo would have obtained two coplanar adjacent squares as well as four coplanar adjacent triangles (see Figure 5, middle). Replacing those two squares by one rectangle and the four triangles by one larger triangle, one gets a solid that indeed resembles the rhombicosidodecahedron, except that its squares are rectangles (see Figure 5, right). Note the solid also recalls the well-known Zome ball (a mathematical toy made by Zometool Inc.), where the rectangles are golden rectangles.
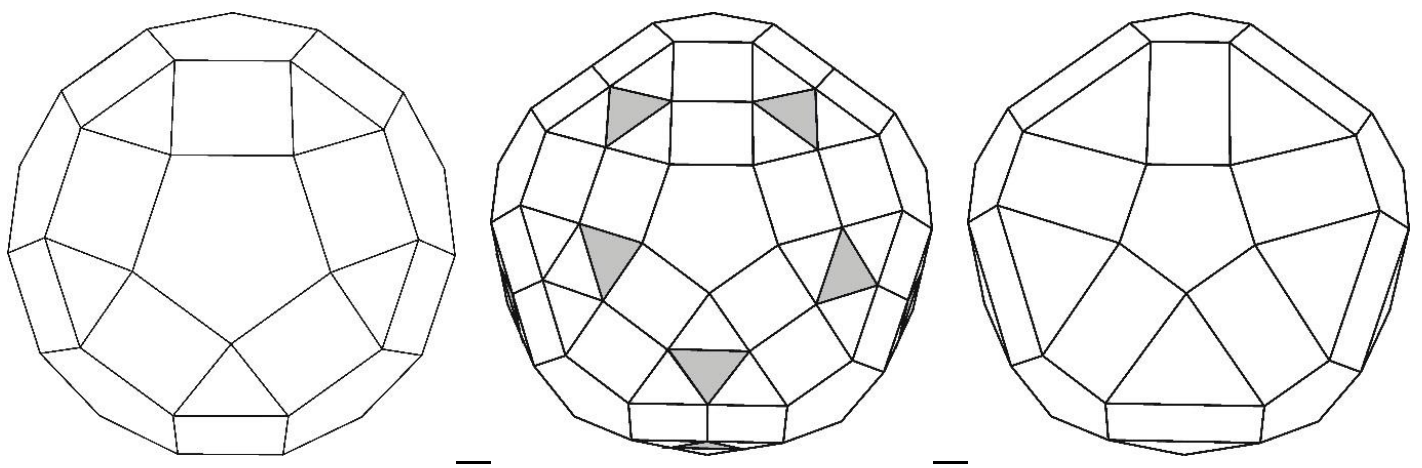

Fig. 5: The rhombicosidodecahedron (left); Leonardo's drawing with all the faces that were elevated (middle); the result is a solid of rhombicosidodecahedron-type when the coplanar faces are replaced by a single face (right).

Assuming that Leonardo also noticed this coplanarity can explain why Leonardo stopped drawing triangles, squares, and pentagons in each decagonal face. However, adding such 'pentagonal cupola' on a truncated dodecahedron was an ingenious and then novel idea. Indeed, a truncated dodecahedron with one pentagonal cupola on it even has a special name today: it is called an "augmented truncated dodecahedron". One can add two or three pentagonal cupolas, but not on adjacent decagons, since otherwise some faces will be coplanar, as we have seen above (see Fig. 6). Today, these solids are called "Johnson solids", after Norman Johnson, who discovered all such 92 strictly convex non uniform solids formed by regular polygons in $1966 .{ }^{12}$ Thus,

11 J.V. Field, 'Rediscovering the Archimedean polyhedra: Piero della Francesca, Luca Pacioli, Leonardo da Vinci, Albrecht Dürer, Daniele Barbaro, and Johannes Kepler', in: Archive for History of Exact Sciences, 50, 3-4 (1997), pp. 241-289.

12 The 92 Johnson solids are not Platonic nor Archimedean as they are not uniform. Like in the case of Archimedean solids, different regular polygons can be used as faces, but now there is no requirement that they are grouped around each vertex in an identical way. Surprisingly, all of the 92 cases are formed only by equilateral triangles, squares, or regular penta- hexa-, octa- or decagons. Typical examples are prisms 
the polyhedron Leonardo proposed on his folio could be called a "(completely) augmented truncated dodecahedron", for lack of a better nomenclature.
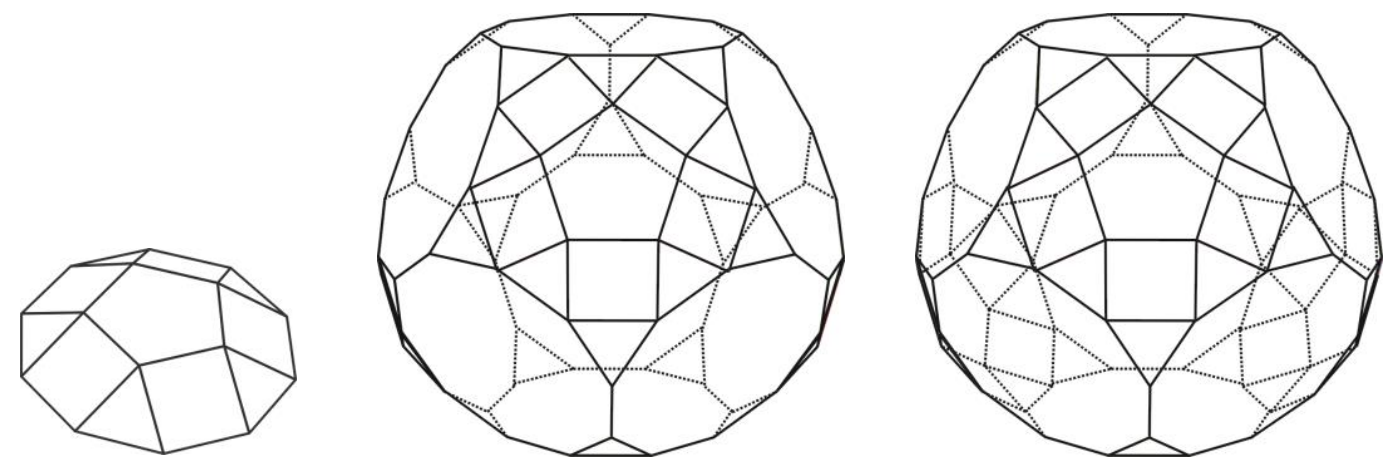

Fig. 6: A pentagonal cupola (left), and a truncated dodecahedron with one cupola (middle) and with three cupolas (right).

Below this "augmented truncated dodecahedron" and to the right of it, Leonardo reminds himself again of the basic Platonic solids, the regular tetrahedron and octahedron. Next, he describes an icosidodecahedron perfectly as having ' 20 triangular and 12 pentagonal' (faces). Below this and without an accompanying diagram, he describes the (not augmented) truncated icosahedron with ' 32 faces: 20 hexagonal and 12 pentagonal'. The icosidodecahedron has pyramids on the equilateral triangles, formed by equilateral triangles. Leonardo drew this solid for Pacioli's Divina in four versions, with only edges or with the faces and with and without pyramids on each face. Going down on the folio one sees a regular dodecahedron with something like a pentagram inscribed in one of its faces. It is possible that this pentagram is only an impression made by triangles inside a pentagon and around a pentagon in the middle. If not, Leonardo perhaps discovered a star polyhedron of the type Johannes Kepler (1571-1630) would mention only much later.

Further down the folio is another dodecahedron, in which Leonardo inscribes a truncated icosahedron, and below it, slightly to the left, yet another icosidodecahedron. It looks like a kind of weaponry, as it has pyramids on its pentagonal faces this time. Such a "pentagonal pyramid" is another example of a Johnson solid if the triangles are equilateral, but that is not necessarily the case in Leonardo's drawing (see Fig. 7). At the far right, there stands a truncated icosahedron of which Leonardo filled some of the hexagonal faces with equilateral triangles. It consists of 12 pentagons and 20 hexagons. Each hexagon (but not the pentagons) is subdivided into 6 triangles, and the total number of these triangles is $6 \times 20=120$, the number above the number 132 in the adjoining text. When the 12 pentagonal faces are added to the already counted 120 triangles, one indeed gets 132 faces.

with pyramids on one of their faces, or pyramids on a square or pentagonal base. The pseudorhombicuboctahedron mentioned in footnote 3 is also more often considered as a Johnson solid rather than as the $14^{\text {th }}$ Archimedean, because of its lack of symmetry. 

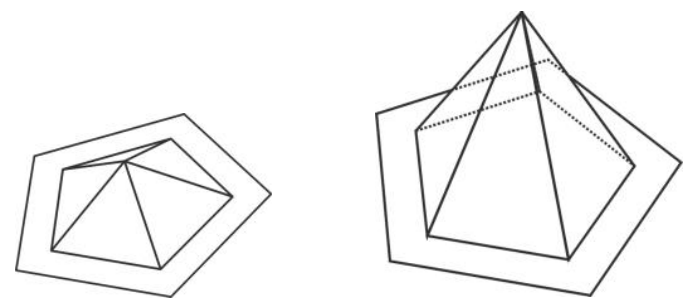

Fig. 7: A pentagonal pyramid with equilateral triangles on the base is a Johnson solid (left), but not when the triangles are isosceles (right); the bases of both were drawn in another larger pentagon, for comparison with Leonardo's drawings.

More remarkable is the solid between the two previously mentioned ones and the lowest one on the folio: a truncated icosidodecahedron with pyramids on its square faces (see Figure 6, left). As was the case above, the underlying solid, now a truncated icosidodecahedron, is not mentioned in Pacioli's Divina. This solid doesn't even figure in Piero's Libellus either. Daniele Barbaro (1513-1570) is known as its rediscoverer, in La pratica della perspettiva $(1568,1569)^{13}$ but that is about 75 years later (the aforementioned truncated dodecahedron is also mentioned in it).
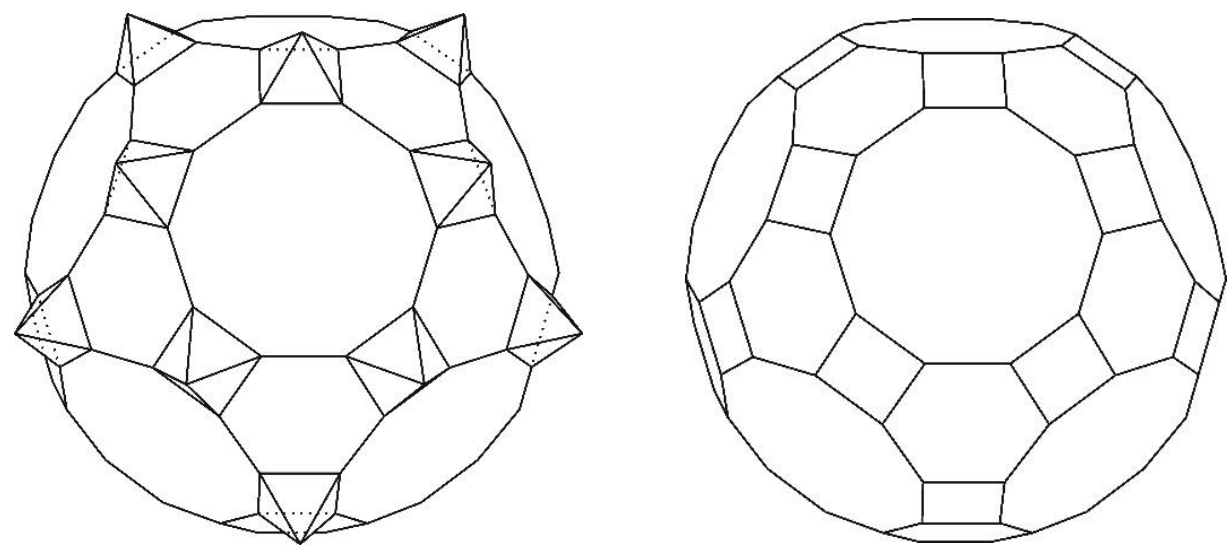

Figure 8: Leonardo's truncated icosidodecahedron with pyramids on the square faces (left) and the underlying truncated icosidodecahedron (right).

\section{Conclusion}

This article elaborated how Leonardo was exercising his drawing skills on this folio while collaborating with Pacioli for the drawings of the Divina Proportione. Through an analysis of the figures, it has been shown that he might not have included the truncated dodecahedron and the truncated icosidodecahedron he drew on this folio in Pacioli's list as he was only asked to draw the solids Pacioli proposed him. After all, it was Pacioli's book and Leonardo was "but" the illustrator. However, a close look at the folio has shown that Leonardo was more than a mere help to Pacioli, as he went on to discover two additional Archimedean polyhedra, as well as a non-Archimedean one, the truncated rhombicuboctahedron, and perhaps even some Johnson solids.

${ }^{13}$ D.M.A. Barbaro, La pratica della perspettiva, Venezia, Camillo \& Rutilio Borgominieri, 1569. 


\section{Keywords}

Luca Pacioli, polyhedra, Archimedean solids, Codex Atlanticus

Dirk Huylebrouck lectured in Congo and Burundi for twelve years, interrupted by assignments in Portugal and at Maryland University Europe. Next, he taught at the Faculty of Architecture of the KU Leuven (Belgium) and edited the column 'The Mathematical Tourist' in the journal The Mathematical Intelligencer. He wrote five books in Dutch, translated into French and/or English): Afrika + wiskunde (2005), De Codes van da Vinci, Bach, pi (2009), België + wiskunde (2009), Wiskunst (2016) and De columns van Professor Pi (2020). Together with Emma Grootveld (Leiden University) and visual artist Rinus Roelofs, he edited the first Dutch translation of De Divina Proportione.

Faculty of Architecture, KU Leuven

Hoogstraat 51

9000 Gent (Belgium)

dirk.huylebrouck@kuleuven.be

Angelo Mingarelli is Professor of Mathematics at Carleton University, Ottawa, Canada. In 2019, he was the chair and organizer of the Leonardo Cinquecento-Carleton University celebrates Leonardo da Vinci, year-long program at Carleton where specialists from around the world were invited on a monthly basis to give talks and share their knowledge of Leonardo da Vinci during the $500^{\text {th }}$ anniversary of his death. He specializes in differential equations and its applications and geometry in its many forms.

School of Mathematics and Statistics

Carleton University

Ottawa, K1S 5B6 (Canada)

angelo@math.carleton.ca

\section{RIASSUNTO}

\section{Il folio 735 di Leonardo}

Nella prefazione a De Divina Proportione (1498 e 1509) Luca Pacioli loda Leonardo per le sue illustrazioni al libro: disegni di prismi, piramidi, cilindri, sfere, i cinque solidi platonici e sei dei tredici solidi archimedei. Sul folio 735 del Codice Atlantico, tuttavia, Leonardo sembra aver disegnato due altri solidi archimedei che sarebbero stati scoperti molto più tardi, oltre a un rombicubottaedro troncato e forse anche alcuni solidi di Johnson. Ė generalmente accettato che Leonardo cominciò a studiare i poliedri nel 1496, dopo aver conosciuto Pacioli alla corte di Milano; di conseguenza è plausibile una datazione del folio tra il 1495 e il 1497. Sebbene De Divina Proportione contenga numerosi disegni di poliedri, Pacioli non incluse nel trattato le scoperte di Leonardo, pur lavorando in stretta collaborazione con il vinciano. 